\title{
Points de vue de professionnels de la santé et des services sociaux sur un programme de visites à domicile destiné aux jeunes mères d'un premier enfant
}

\author{
S.-A. Li, M. Sc. (1, 2); S. M. Jack, Ph. D. (1, 2, 3); A. Gonzalez, Ph. D. (2, 4); E. Duku, Ph. D. (2, 4); \\ H. L. MacMillan, M.D. $(1,2,4,5)$
}

Cet article a fait l'objet d'une évaluation par les pairs.

Diffuser cet article sur Twitter

\section{Résumé}

Introduction : On sait peu de choses sur ce que pensent les professionnels de la santé et des services sociaux des programmes à long terme de visites à domicile pour les nouvelles mères à faible revenu. Cette étude fait état des expériences et des points de vue de fournisseurs de services communautaires qui orientent les mères participant au Nurse-Family Partnership (NFP) - un programme de visites à domicile par une infirmière auprès de cette population cible - vers le programme lui-même ou qui interviennent directement dans le cadre de celui-ci.

Méthodologie : L'étude s'est déroulée en deux phases. La phase I a consisté en une analyse secondaire de données qualitatives issues d'un échantillon de 24 entrevues individuelles dirigées avec des fournisseurs de soins communautaires, cette démarche s'inscrivant dans le cadre d'une étude de cas plus vaste réalisée à Hamilton (Ontario, Canada) et destinée à examiner les moyens à mettre en œuvre pour accroître la recevabilité du NFP. La phase II, à laquelle ont pris part 4 participants, a consisté en une description qualitative des 3 thèmes relevés lors de la phase $\mathrm{I}$, thèmes qui ont été approfondis au moyen d'entrevues individuelles semi-structurées menées auprès de certains fournisseurs de soins de santé et de services sociaux communautaires.

Résultats : Le NFP est perçu dans l'ensemble comme un programme comblant une lacune importante dans les services dispensés aux nouvelles mères. Des échanges fréquents entre le NFP et les organismes communautaires offrant déjà des services à ces mères pourraient contribuer à en améliorer le processus d'orientation, à éviter leur chevauchement et à en faciliter l'accès. Ces résultats contribuent à mieux définir les composantes nécessaires au succès de l'intégration d'un programme de visites à domicile au réseau déjà en place de services communautaires.

Conclusion : Le rôle des programmes de visites à domicile ne doit pas être envisagé isolément. $\mathrm{Au}$ contraire, le potentiel de ceux-ci est optimisé par la collaboration et l'échange d'information avec d'autres organismes afin d'offrir de meilleurs services aux mères d'un premier enfant.

Mots-clés : visites à domicile, intervention précoce, éducation des parents, mères, NurseFamily Partnership, professionnels du milieu communautaire

\section{Principales constatations}

- Les fournisseurs de soins de santé et de services sociaux communautaires ont confirmé la valeur ajoutée apportée par le programme NurseFamily Partnership (NFP) aux services communautaires déjà en place.

- Ils préconisent une collaboration accrue et plus fréquente avec le NFP de façon à former un réseau intégré de services pour les jeunes mères défavorisées et à faciliter l'accès de ces dernières aux services communautaires.

\section{Introduction}

Les femmes enceintes dont les ressources financières, psychologiques et personnelles sont limitées vivent une grossesse davantage à risque, en particulier celui de donner naissance à un enfant prématuré ou de faible poids ${ }^{1,2}$. L'accès aux services sociaux et de santé communautaires et leur utilisation par les femmes enceintes défavorisées et les familles ayant de jeunes enfants est facilité par l'existence de visites à domicile avant la naissance ou pendant la petite enfance ${ }^{3,4}$. Au Canada, on offre à la fois des services universels (non ciblés) de visites à domicile aux nouveaux ou futurs parents et des visites plus soutenues à certaines catégories de population, habituellement les familles dont les enfants risquent davantage d'être en mauvaise santé ou d'avoir des lacunes sur le plan du développement social ou émotionnel ${ }^{5}$.

Rattachement des auteurs :

1. Département d'épidémiologie clinique et de biostatistique, Université McMaster, Hamilton (Ontario), Canada

2. Offord Centre for Child Studies, Université McMaster, Hamilton (Ontario), Canada

3. School of Nursing, Université McMaster, Hamilton (Ontario), Canada

4. Département de psychiatrie et de neurosciences comportementales, Université McMaster, Hamilton (Ontario), Canada

5. Département de pédiatrie, Université McMaster, Hamilton (Ontario), Canada

Correspondance : Shelly-Anne Li, Offord Centre for Child Studies, 1280, rue Main Ouest, Hamilton (Ontario) L8S 4K1; tél. : 416-662-2232; courriel : shellyanne.li@gmail.com 
Le Nurse-Family Partnership (NFP) est un programme fondé sur des données probantes qui s'inspire des théories sur l'« écologie humaine " ${ }^{6}$, l'auto-efficacité ${ }^{7}$ et l'attachement humain ${ }^{8}$. Au cours des 35 dernières années, il a fait l'objet d'évaluations approfondies reposant sur une série de trois essais cliniques randomisés (ECR $)^{9-11}$. Sa mise en œuvre a commencé dès que l'efficacité des interventions visant à améliorer la grossesse ainsi que la santé et le bienêtre psychosocial de la mère et de l'enfant a été prouvée. Il touche à l'heure actuelle les familles admissibles de 43 États américains ainsi que des Îles Vierges américaines ${ }^{11-13}$.

Le NFP a élaboré 18 éléments de modèle pour guider les organismes mettant en œuvre ses interventions. Ces éléments assurent l'obtention de résultats comparables à ceux des trois ECR à la base à l'extension actuelle du programme ${ }^{14}$. Deux d'entre eux renvoient expressément à l'importance du partenariat avec d'autres organismes, au soutien et à la reconnaissance communautaires et au niveau de mobilisation communautaire requis pour le succès de cette mise en œuvre ${ }^{15}$. L'établissement d'alliances entre les intervenants communautaires et les organismes relevant de divers secteurs pour promouvoir et maintenir la santé des collectivités est une stratégie importante pour combler les besoins de ces collectivités en matière de santé ${ }^{16}$. Les intervenants à domicile nouent ainsi des partenariats avec d'autres professionnels de la santé et des services sociaux (p. ex. les médecins de première ligne, les pédiatres et les travailleurs sociaux), qui fournissent une aide vitale et une éducation de base aux familles exposées à des risques élevés au sein d'une collectivité $\mathrm{e}^{17-19}$. On estime que ces partenariats améliorent le respect des mesures de prévention et des traitements par les familles ${ }^{20}$. Dans ce cadre, il est fondamental de comprendre le point de vue des professionnels de la santé formant ce réseau de soutien élargi dans lequel s'inscrit le NFP, car le soutien communautaire est un élément déterminant du succès de la mise en œuvre du programme.

Le NFP a déjà été évalué au Canada, plus précisément à Hamilton (Ontario) ${ }^{21,22}$, et un ECR est en cours en Colombie-Britannique pour mesurer son efficacité. D'après les enquêtes approfondies qui ont été réalisées, les infirmières de la santé publique (ISP) au
Canada ainsi que les clients qui bénéficient de l'intervention et leurs familles ont bien accueilli les éléments de modèle du NFP et les visites à domicile $e^{21-24}$. Il restait à examiner le point de vue des professionnels de la santé et des services sociaux qui interviennent dans le cadre du NFP : notre étude remédie à cette lacune en sondant leur opinion sur les facteurs qui influencent la bonne réception de ces programmes de visites à domicile. Elle prend en compte les points de vue des professionnels susceptibles de renseigner les administrateurs des services de santé sur la mesure dans laquelle la collectivité est prête à accueillir un nouveau programme de visites à domicile fondé sur des données probantes et s'intégrant au système de santé canadien déjà en place.

\section{Méthodologie}

Cette étude qualitative a comporté deux phases. La phase I a consisté en une analyse secondaire des données tirées d'une étude de cas qualitative ${ }^{21}$ réalisée à Hamilton (Ontario) et visant à évaluer si le NFP était accueilli favorablement par les nouvelles mères à faible revenu et leurs familles, les ISP et les intervenants communautaires, notamment les professionnels de la santé et des services sociaux. Les données utilisées pour cette analyse secondaire étant issues de questions posées par les chercheurs au cours de l'analyse primaire, les réponses étaient susceptibles de ne pas refléter adéquatement les questions de recherche définies dans notre étude ${ }^{25}$. La phase II a consisté, pour surmonter cet obstacle, à mener d'autres entrevues individuelles semi-structurées auprès de professionnels du milieu communautaire. Ces entrevues font également office de suivi pour l'élaboration et la confirmation des constatations de la phase I : la phase II a en effet été entreprise aussi pour déterminer si les constatations recueillies lors de la phase I en 2009 étaient toujours valables en 2014.

\section{Échantillonnage}

Pour la phase I, nous avons constitué un sous-ensemble de 24 entrevues individuelles avec des intervenants communautaires (professionnels de la santé et des services sociaux). Le tableau 1 présente les caractéristiques de cet échantillon.

Nous avons procédé à un échantillonnage dirigé de ces participants pour la phase II en vue de sélectionner ceux qui étaient susceptibles de fournir à nos questions de

TABLEAU 1

Professionnels de l'échantillon de la phase I

\begin{tabular}{lc} 
Activité professionnelle & $\mathbf{n}(\%)$ \\
\hline Fournisseurs de soins de première ligne (chefs d'équipe, travailleurs de soutien) & $5(20,8)$ \\
Professionnels de la santé (médecins, infirmiers autorisés, infirmières autorisées) & $3(12,5)$ \\
Praticiens en services sociaux (travailleurs sociaux, agents de liaison avec les écoles) & $6(25,0)$ \\
Responsables (directeurs, superviseurs, gestionnaires) & $6(25,0)$ \\
Professionnels de la protection de l'enfance & $3(12,5)$ \\
Personnel de soutien du NFP & $1(4,2)$ \\
\hline Titre professionnel & $\mathbf{n}(\%)$ \\
\hline Travailleur social autorisé, travailleuse sociale autorisée & $17(70,8)$ \\
Médecin & $2(8,3)$ \\
Infirmier autorisé, infirmière autorisée & $2(8,3)$ \\
Autre diplôme & $2(8,3)$ \\
Non fourni & $1(4,2)$ \\
\hline Autres données & Moyenne (é.-t.) \\
\hline Âge (ans) & $51,8(5,3)$ \\
Nombre d'années dans la profession & $18,1(0,5)$ \\
Nombre d'années d'expérience dans le poste actuel & $7,8(5,5)$ \\
Nombre de femmes enceintes à faible revenu ayant bénéficié & $96,6(172,5)$ \\
d'une intervention au cours de la dernière année & \\
\hline
\end{tabular}

Abréviations : é-t, écart-type; NFP, Nurse-Family Partnership. 
recherche des réponses enrichissantes. Cet échantillon ciblait à la fois des professionnels de la Société d'aide à l'enfance (SAE) pouvant s'exprimer sur le rôle du NFP dans le contexte des services de protection de l'enfance et des professionnels des services de santé publique de Hamilton (SSPH) susceptibles de fournir des renseignements détaillés sur le programme de visites à domicile du NFP à Hamilton. Sachant, grâce à la phase I, que de nombreux fournisseurs de soins communautaires connaissaient l'influence du NFP sur le nombre de dossiers adressés à la SAE et sur le délai de fermeture des dossiers de la SAE, nous avons exclu de l'échantillon les professionnels n'étant pas pas directement au courant de la mise en œuvre du NFP à Hamilton.

Nous avons communiqué au total avec dix professionnels des services sociaux et de santé (trois du SSPH et sept du SAE) que nous avons considérés comme ayant une connaissance directe du NFP, soit huit ayant participé à l'étude primaire et deux autres sélectionnés par échantillonnage dirigé*. Sur les huit ayant participé à l'étude primaire, quatre ont répondu au courriel d'invitation envoyé par le chercheur principal et trois ont accepté de participer. L'une des deux personnes sélectionnées par échantillonnage dirigé a également accepté. En tout, ce sont donc quatre professionnels des services sociaux et de santé qui ont accepté de participer à notre étude.

\section{Participants}

Les quatre professionnels retenus (3 femmes et 1 homme) participaient au NFP depuis sa mise en œuvre à Hamilton : ils étaient donc en mesure de fournir des renseignements contextuels riches. Ils travaillaient pour leurs organismes respectifs ( 3 pour le SAE et 1 pour le SSPH) depuis en moyenne 19 ans (écarttype de 6 ans) et y occupaient leurs fonctions actuelles depuis en moyenne 12 ans (écarttype de 4 ans). Deux détenaient un baccalauréat et deux une maîtrise en travail social.

\section{Collecte de données}

Les participants devaient signer un formulaire de consentement éclairé avant chaque entrevue. Les données ont été recueillies sur support numérique au moyen d'entrevues individuelles semi-structurées d'environ 60 minutes. Pour mieux apprécier et comprendre les expériences et les points de vue des professionnels du milieu communautaire, un guide d'entrevue a été élaboré, essentiellement à partir des thèmes relevés pendant la phase I (voir tableau 2, les guides d'entrevue étant fournis sur demande par les auteurs). L'analyse et la collecte des données se déroulant en parallèle, les questions ont été modifiées au fur et à mesure dans le guide d'entrevue pour mieux traiter des différents thèmes.

\section{Gestion des données}

Une fois transcrites, les entrevues ont été anonymisées et importées dans NVivo 10 (QSR International Pty Ltd, Doncaster, Victoria $^{26}$. L'utilisation de NVivo a aussi permis de suivre rigoureusement le processus de recherche et d'améliorer ainsi le caractère vérifiable et la crédibilité des constatations.

\section{Analyse des données}

À notre connaissance, aucune étude antérieure n'avait exploré les facteurs qui influencent la réception d'un programme d'intervention précoce chez les fournisseurs de soins de santé et de services sociaux. Nous avons donc appliqué une approche inductive classique d'analyse de contenu aux données qualitatives des phases I et II, puis nous avons caractérisé les codes émergents en fonction de l'information transmise par les participants à l'étude. Nous n'avons pas imposé de catégories préétablies ni de point de vue théorique. Nous avons dégagé l'information à partir des points de vue des répondants tels qu'ils ont été générés avec cette approche classique ${ }^{27}$.

Pour la phase I, nous avons commencé l'analyse par un codage ouvert, qui a consisté à souligner dans les transcriptions les mots les plus représentatifs des idées fondamentales exprimées par les répondants au sujet du programme de visites à domicile du NFP. Des codes
TABLEAU 2

\section{Thèmes, catégories et codes de la phase I}

\begin{tabular}{|c|c|c|}
\hline Thème & Catégorie & Codes \\
\hline \multirow{5}{*}{$\begin{array}{l}\text { Le NFP en tant que programme d'éducation } \\
\text { structuré, fondé sur des données probantes et } \\
\text { destiné aux femmes qui deviennent mères } \\
\text { pour la première fois }\end{array}$} & \multirow[t]{3}{*}{ Éléments du NFP } & Structure et intensité du programme \\
\hline & & $\begin{array}{l}\text { Le NFP en tant qu'intervention } \\
\text { fondée sur des preuves }\end{array}$ \\
\hline & & Préparer les clientes à la maternité \\
\hline & \multirow[t]{2}{*}{ Rôle des ISP } & $\begin{array}{l}\text { Relations ISP-cliente en tant } \\
\text { qu'aspect essentiel du NFP }\end{array}$ \\
\hline & & $\begin{array}{l}\text { Expertise des ISP et image } \\
\text { professionnelle }\end{array}$ \\
\hline \multirow[t]{4}{*}{$\begin{array}{l}\text { Le NFP en partenariat avec d'autres organismes } \\
\text { communautaires }\end{array}$} & & $\begin{array}{l}\text { Les ISP, courroie de transmission avec } \\
\text { les services communautaires pour les } \\
\text { clientes }\end{array}$ \\
\hline & & $\begin{array}{l}\text { Rôle de soutien communautaire pour } \\
\text { assurer la continuité et le } \\
\text { développement du NFP }\end{array}$ \\
\hline & \multirow{2}{*}{$\begin{array}{l}\text { Collaboration } \\
\text { entre les } \\
\text { organismes et le } \\
\text { NFP }\end{array}$} & $\begin{array}{l}\text { Préférence pour une poursuite de la } \\
\text { collaboration avec le NFP }\end{array}$ \\
\hline & & $\begin{array}{l}\text { Préférence pour une communication } \\
\text { ininterrompue avec les ISP du NFP }\end{array}$ \\
\hline \multirow[t]{3}{*}{ Le NFP en contexte communautaire } & \multirow[t]{3}{*}{ Retombées du NFP } & $\begin{array}{l}\text { Offre de services et de soutien visant } \\
\text { à combler une lacune en matière de } \\
\text { besoins }\end{array}$ \\
\hline & & $\begin{array}{l}\text { Problème de chevauchement avec les } \\
\text { services communautaires déjà en place }\end{array}$ \\
\hline & & Services de protection de l'enfance \\
\hline
\end{tabular}

Abréviations : ISP, infirmière de la santé publique; NFP, Nurse-Family Partnership.

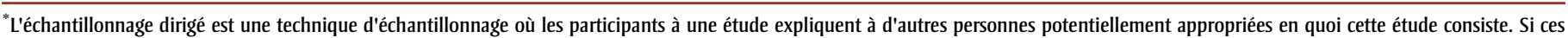
dernières sont intéressées et répondent aux critères de l'étude, elles peuvent elles aussi y prendre part. 
(essentiellement des « étiquettes » assignées à des segments de texte formant des unités de sens) ont émergé. Nous avons créé une liste de ces codes préliminaires à partir du codage ouvert de cinq transcriptions, puis nous avons élaboré un guide pour le codage des autres entrevues. Ce guide a été revu et peaufiné au fur et à mesure que de nouveaux codes et idées ont émergé des transcriptions. Certaines catégories ont ainsi été regroupées tandis que d'autres ont été divisées en souscatégories, en fonction des liens entre les différents codes. Différents thèmes sont ressortis du sens sous-jacent de ces catégories. Nous avons suivi une méthode similaire pour l'analyse des données de la phase II: nous avons utilisé les codes élaborés à la phase I pour amorcer l'analyse puis nous avons enrichi le guide des codes au fur et à mesure de l'émergence de nouveaux codes et idées lors de la phase II.

Nous avons appliqué les quatre critères recommandés par Lincoln et $\mathrm{Guba}^{28}$ crédibilité, transférabilité, fiabilité et cohérence interne - pour garantir la rigueur de l'étude. La crédibilité a été assurée par les commentaires des pairs et par les vérifications effectuées par l'équipe. Un second codeur (MT) a codé un sous-ensemble de transcriptions (quatre transcriptions de la phase I et une de la phase II) puis nous avons comparé ces codes à ceux du premier codeur afin d'en assurer la fiabilité. Pour garantir la transférabilité, nous avons décrit en détail le contexte de la recherche et les caractéristiques des répondants. Enfin, le maintien d'une piste de vérification et de notes de terrain a garanti la cohérence interne.

Le Hamilton Integrated Research Ethics Board (comité d'éthique de la recherche intégrée de Hamilton) a approuvé l'étude.

\section{Résultats}

Lors de la phase I (analyse secondaire des entrevues individuelles avec les fournisseurs de services sociaux et de santé communautaires), nous avons dégagé trois thèmes principaux: (1) le NFP en tant que programme d'éducation structuré, fondé sur des données probantes et destiné aux femmes qui deviennent mères pour la première fois, (2) le NFP en partenariat avec d'autres organismes communautaires et (3) le NFP en contexte communautaire (tableau 2).

Ces thèmes ont été explorés lors de la phase II au moyen des entrevues auprès des professionnels des SSPH et du SAE de la municipalité d'Hamilton (Ontario) ayant une expérience et des connaissances approfondies de la collaboration entre les ISP, les superviseurs et les clients du NFP.

Le NFP en tant que programme d'éducation structuré, fondé sur des données probantes et destiné aux femmes qui deviennent mères pour la première fois

Les fournisseurs de services sociaux et de santé interrogés ont mentionné que le NFP comblait une lacune importante dans les services offerts aux nouvelles mères à faible revenu de Hamilton. Tous sans exception ont exprimé leur satisfaction à l'égard de la structure du programme, de son intensité sur le plan de la préparation des clientes à la maternité, de sa base solide de données probantes, de l'apport de l'expertise des ISP au programme de visites à domicile et enfin des liens étroits noués entre chaque cliente et son ISP.

\section{Éléments du NFP}

Les répondants ont relevé plusieurs spécificités du NFP : visites à domicile débutant tôt au cours de la grossesse, visites fréquentes des infirmières et fait que les familles bénéficient du programme jusqu'à ce que l'enfant ait deux ans. En outre, elles ont estimé que la clarté avec laquelle les objectifs de chaque visite étaient définis conformément aux lignes directrices constituait un atout majeur du NFP et en faisait un programme original si on le comparait aux autres programmes communautaires de visites à domicile. Lors de chaque visite à domicile, l'ISP aborde six aspects essentiels : la santé personnelle, la salubrité de l'environnement, le rôle de la mère, le développement de au cours de la vie, la famille et les amis et enfin les services sociaux et de santé. Les répondants ont aussi souligné que le programme avait tendance à encourager des relations parent-enfant positives et à favoriser l'acquisition de compétences parentales solides tout en parvenant à un équilibre optimal entre l'intensité du programme et la fréquence des visites à domicile planifiées. L'un des fournisseurs de première ligne travaillant dans une maison de maternité a ainsi témoigné :

[traduction] Ce que j'apprécie le plus, c'est à quel point le programme est intense et débute tôt au cours de la grossesse. Vous savez, je pense que cela amène les futures mères à commencer à penser à leur rôle de parent et à ce genre de choses... J'aime aussi le fait qu'après la naissance de l'enfant, les visites continuent une fois par semaine, pendant six semaines... parce que c'est... disons une période où le risque est élevé. Je pense que c'est une force du programme, cela et aussi le fait qu'il se poursuive pendant deux ans... C'est quelque chose de très intense... enfin, c'est mon opinion. (S523)

La solidité des résultats associés à la participation des clients au NFP a aussi suscité des réactions positives chez les responsables des organismes et les fournisseurs de soins de première ligne interrogés. Ils ont considéré que cette participation était importante et son efficacité prouvée. Les répondants connaissaient de manière générale les données provenant des États-Unis, notamment les résultats de l'extension du programme du NFP. Un professionnel de la SAE a ainsi noté :

[traduction] En ce qui concerne ce programme, j'ai été très impressionné... De toute évidence, les résultats obtenus aux États-Unis, si l'on examine les effets et les résultats à long terme... sont très impressionnants. (S509)

Les participants à l'étude ont décrit le NFP comme très bénéfique pour les nouvelles mères à faible revenu. Du fait qu'elles établissent des liens avec la cliente avant la naissance du bébé, les ISP sont considérées comme étant dans une position idéale pour réduire les facteurs de risque connus relativement à la santé prénatale et néonatale, de même que pour aider la cliente à se préparer à son nouveau rôle de 
parent. L'un des fournisseurs de soins de première ligne a ainsi décrit les avantages des aspects éducatifs du programme :

[traduction] Ah, et les aspects du programme qui touchent l'éducation et les activités éducatives pratiques aident énormément les filles... c'est vraiment important que quelqu'un soit là pour les aider avec ce genre de choses... leur donner... une chance de parler de leurs inquiétudes au sujet de leur rôle de parent ou du fait de devoir prendre soin de leur enfant. (S521)

Ce commentaire renvoie à une composante essentielle du NFP : l'établissement d'une relation thérapeutique entre la cliente et l'ISP, en particulier lorsque l'ISP devient une personne à qui parler de ses soucis quotidiens. L'ISP joue un rôle essentiel non seulement dans l'éducation des clientes sur des questions de santé et dans l'enseignement de pratiques parentales positives, mais aussi dans leur responsabilisation, en renforçant leur confiance en elles-mêmes et leur estime de soi ainsi qu'en intégrant à leur vie des activités positives et constructives.

\section{Rôle des ISP}

Les répondants percevaient les interactions individuelles régulières entre l'ISP et la cliente comme essentielles à l'établissement d'une relation de confiance, relation susceptible de durer deux ans et demi. Un travailleur social s'exprime par exemple ainsi :

[traduction] Je pense que c'est certainement quelqu'un qu'elles n'hésitent pas à appeler chaque fois qu'elles ont des questions ou des inquiétudes... je crois comprendre que les clientes connaissent le numéro de cellulaire de leur ISP. Il est plutôt rare de n'avoir qu'à prendre le téléphone pour avoir tout de suite au bout du fil un professionnel de la santé... Je pense que les jeunes mères doivent pouvoir compter sur quelqu'un en qui elles ont confiance. (S511)

Tous les répondants étaient d'avis que les ISP apportent aux clientes du NFP des connaissances et des compétences réelles sur les questions de santé. Ils percevaient les ISP comme ayant une meilleure réputation et une image plus positive que celle d'autres professionnels du milieu communautaire œuvrant auprès des jeunes mères à faible revenu, notamment les travailleurs sociaux et les professionnels de la SAE ou des services de soutien aux parents. Un travailleur social a ainsi opposé l'image publique des ISP et celle des travailleurs sociaux et des professionnels de la SAE :

[traduction] Je pense que vous savez que les gens se sentent souvent plus nerveux lorsqu'ils savent que... s'ils savent que c'est un travailleur social. Il m'est souvent plus facile de vendre un programme si je dis qu'il est volontaire, que c'est une infirmière qui rend visite à des gens de tous les milieux, que ce n'est pas quelqu'un qui vient «contrôler ce que vous faites ». C'est un peu plus ce que ressentent les gens lorsqu'il s'agit d'un professionnel de la SAE ou des services de soutien aux parents. (S511)

\section{Le NFP en partenariat avec d'autres organismes communautaires}

L'ISP, courroie de transmission pour les services communautaires destinés aux clientes

Les fournisseurs de soins communautaires voient les ISP comme des courroies de transmission pour les services communautaires offrant un soutien aux jeunes mères à faible revenu, que ce soit l'aide pour se loger, pour les études ou pour les soins de santé. Ils ont souligné l'importance de disposer de programmes collaboratifs et insisté sur la valeur d'une approche bidirectionnelle dans la promotion des services communautaires.

Les répondants ont aussi souligné le rôle à jouer par le soutien communautaire dans le maintien et l'essor du NFP. Par exemple, pour l'un des fournisseurs de services sociaux, le NFP doit s'insérer dans un processus continu de soutien aux mères à risque élevé, étant donné que leurs problèmes de logement et d'emploi ainsi que les questions de santé mentale ne sont vraisemblablement pas tous résolus lorsque le programme du NFP prend fin. Le NFP est donc bien placé pour préparer les autres organismes déjà chargés d'épauler ces jeunes mères ou à la veille de le faire.
Pour former une infrastructure de soutien concrète et efficace pour cette population cible, resterait donc à renforcer la collaboration entre le NFP et les autres organismes communautaires. L'un des fournisseurs de soins communautaires a ainsi parlé de la collaboration comme d'un moyen de prévenir le chevauchement dans les services offerts aux clientes:

[traduction] C'est juste une question de liaison et, bien sûr, de collaboration, pour s'assurer qu'il n'y a pas de chevauchement. Je pense que c'est cela qui est vraiment important, et c'est ce que j'essaie de faire lorsqu'il y a un certain nombre d'organismes différents, en particulier lorsqu'il y a un NFP... unissons-nous ... (S519)

Positionnement en faveur d'une communication ininterrompue avec les ISP du NFP

Les fournisseurs de soins ont souligné les avantages potentiels d'une communication renforcée avec les ISP du NFP, notamment une meilleure orientation, la capacité de renforcer l'appui de la collectivité envers le NFP et une meilleure connaissance des objectifs de ce dernier. Pour l'un d'entre eux, la planification de réunions avec le NFP aiderait les fournisseurs de soins communautaires à se maintenir au courant des progrès du NFP au sein de la collectivité, ce qui aiderait les organismes pour lesquels ils travaillent à soutenir plus efficacement le NFP, en particulier dans l'orientation vers les ressources :

[traduction] Je pense à des réunions de temps en temps, pour nous tenir à jour, pour nous informer de ce qui se passe afin que nous puissions appuyer le programme. Ainsi, vous savez, c'est... ce qui se passe, c'est que lorsque vous savez en quoi quelque chose consiste, vous êtes d'autant plus apte à bien cibler la clientèle qui peut en bénéficier. (S503)

\section{Le NFP en contexte communautaire}

Le NFP est perçu comme un programme ayant une incidence positive sur les organismes communautaires sans pour autant perturber la logistique des services déjà en place. Les fournisseurs de soins 
communautaires ont le sentiment que le NFP comble à Hamilton une lacune dans les services offerts aux jeunes mères d'un premier enfant défavorisées. Beaucoup ont aussi fait état de l'influence du NFP sur les services de protection de l'enfance, notamment la fermeture plus rapide des dossiers de la SAE, la réduction du nombre de dossiers ouverts à la SAE et l'amélioration $\mathrm{du}$ processus de planification visant à assurer la sécurité des familles à risque élevé. Cependant, certains fournisseurs se sont dits préoccupés par le chevauchement avec les services déjà en place, et certains ont comparé le NFP aux SSPH.

\section{Influence du NFP sur les services communautaires déjà en place}

Lorsque l'on a demandé aux répondants quel effet le NFP avait sur les services déjà en place, tous ont affirmé qu'il ne constituait pas une gêne dans la manière dont les interventions se faisaient, mais qu'il avait plutôt pour effet d'accroître les services offerts aux nouvelles mères. Ils ont fait état d'une influence positive directe et indirecte (par l'entremise des clientes) des ISP sur leurs services. Pour reprendre les propos d'un médecin :

[traduction] Oui, je suis très enthousiaste à l'égard de ce programme. En tant que médecin de famille, il me permet de gagner du temps, et je sais que je peux diriger ma patiente vers des ressources qui, je l'espère, pourront contribuer à ce qu'elle garde son enfant et soit une meilleure mère... En fait, pour être parfaitement honnête, je vais vous dire qu'aucun médecin de famille, quel que soit son degré de sollicitude envers ses patients, n'a le temps de faire ce que font les infirmières. (S514)

\section{Influence du NFP sur les services de protection de l'enfance}

De nombreux répondants ont fait état de retombées spécifiques du NFP sur les services de protection de l'enfance (notamment la SAE) - ce qui justifie une description distincte de celle des résultats portant sur les autres services communautaires. Les travailleurs de la SAE ont avoué se sentir plus confiants lorsqu'un autre professionnel de la santé travaillait en proche collaboration avec leurs clientes. Ils ont également souligné qu'ils étaient convaincus que les ISP allaient faire part de leurs préoccupations concernant leurs clientes à la SAE. Un autre participant a estimé que le NFP constituait un «travail plus intense» et qu'il avait «plus confiance dans le réglement du dossier [SAE] » lorsqu'une ISP rendait régulièrement visite à la cliente. Ce participant a précisé :

[traduction] J'ignore quelle tournure aurait pu prendre ce dossier particulier s'il n'y avait pas eu une autre personne [l'ISP] pour aider à organiser les rencontres que nous avons eues, pour aider à ce que quelqu'un d'autre suive la situation de près, et également pour m'informer sur ce qui se passait réellement, pas vrai? Je crois que cela a vraiment contribué à me fournir une meilleure idée de la situation et à faciliter ma planification, puis à offrir de meilleures conditions et un meilleur plan pour le bébé et pour la mère. (S515)

Les professionnels de la SAE ont aimé travailler avec les ISP du NFP pour leurs clientes communes, et ils nous ont souvent décrit leurs expériences positives et l'appui précieux qu'ils ont reçu de la part des ISP. Cependant, certains obstacles à une collaboration transparente sont bien réels. Une professionnelle de la SAE a par exemple relaté son expérience avec une ISP alors qu'elles travaillaient «ensemble, côte à côte ». Elle a parlé de la difficulté à comprendre leurs rôles respectifs, notamment lorsque son rôle en tant travailleuse de la SAE a changé après qu'il a été statué qu'un enfant avait besoin de protection (au sens de la Loi sur les services à l'enfance et à la famille de l'Ontario ${ }^{29}$ ) et que la SAE a dû intervenir pour la cliente :

[traduction] Je dois avouer que parfois, c'était... Je ne savais pas si c'était parce qu'elle ne comprenait pas notre rôle et peut-être que je ne comprenais pas son rôle comme j'aurais dû, pour qu'on collabore mieux... lorsque le bébé est né et que c'est devenu un dossier de protection... il a fallu se préoccuper de la sécurité et de la protection de l'enfant. Nos rôles ont alors changé. Lorsque j'ai travaillé avec l'ISP du NFP avec cette mère avant la naissance du bébé, c'était très différent et plus facile de la suivre... mais une fois que c'est devenu un cas de protection, j'ai eu à intervenir. J'ai eu à mettre en place les services offerts par la Société pour cette mère parce que c'était devenu un cas de protection et qu'il y avait des problèmes... (S516)

\section{Synthèse des résultats}

L'intégration des résultats des deux phases de l'étude nous a permis d'approfondir les constatations de l'analyse secondaire des données et de répondre à certaines questions restées en suspens. Les constatations de la phase II de l'étude (reflétant les perceptions actuelles sur la prestation du NFP) ont largement concordé avec celles de la phase I (remontant à 2009), comme l'illustre le tableau 3 qui en rassemble les éléments communs. Les données de la phase II nous ont en outre renseigné sur l'état actuel du programme du NFP, notamment dans les domaines ayant nécessité un examen plus approfondi, et nous en ont appris davantage sur les défis auxquels le NFP est confronté (tableau 4).

\section{Analyse}

Nos résultats offrent une information précieuse sur la réception du NFP dans une collectivité canadienne. Les points de vue des fournisseurs de soins de santé et de services sociaux communautaires confirment les descriptions des expériences vécues par les jeunes mères défavorisées ayant un premier enfant qui ont participé au NFP à Hamilton (Ontario) ${ }^{28}$. Celles-ci ont notamment jugé que les ISP étaient des spécialistes crédibles en matière de santé en même temps que des amies leur apportant un soutien et en qui elles pouvaient avoir confiance. De plus, les mères ayant participé à l'étude ont fait état $\mathrm{du}$ caractère stimulant des relations qu'elles ont entretenues avec les ISP et elles ont estimé que ces dernières défendaient bien leurs droits auprès des organismes communautaires.

Après presque six mois de travail avec le NFP à Hamilton, les professionnels de la SAE et les ISP semblent avoir adopté plusieurs des stratégies proposées par les fournisseurs de soins dans la phase I pour promouvoir la collaboration entre les organismes. Ces stratégies consistent à (1) fournir au début de la 
TABLEAU 3

Sommaire des constatations concordantes entre les entrevues de la phase I et celles de la phase II

\begin{tabular}{|c|c|}
\hline Thème & Résumé \\
\hline $\begin{array}{l}\text { Le NFP en tant que programme d'éducation structuré, } \\
\text { fondé sur des données probantes et destiné aux femmes qui } \\
\text { deviennent mères pour la première fois }\end{array}$ & $\begin{array}{l}\text { Les répondants ont fait état des commentaires positifs des clientes au sujet du NFP. Les jeunes mères } \\
\text { semblent très satisfaites des produits du programme. } \\
\text { L'idée d'établir une relation à long terme avec la famille est toujours considérée comme le principal } \\
\text { élément du NFP. } \\
\text { Les répondants ont parlé des avantages que procure le NFP à la mère et à l'enfant. } \\
\text { Le besoin de services d'interprétation pour mieux dépister les jeunes mères est encore présent. }\end{array}$ \\
\hline $\begin{array}{l}\text { Le NFP en partenariat avec d'autres organismes } \\
\text { communautaires }\end{array}$ & $\begin{array}{l}\text { Les répondants ont suggéré une communication accrue et des moyens d'échanger fréquemment } \\
\text { l'information (p. ex., inviter les ISP à visiter les organismes pour sensibiliser les employés à la } \\
\text { situation des jeunes mères qui ont un premier enfant et à leurs expériences avec elles, ou à fournir } \\
\text { des mises à jour rapides par téléphone). }\end{array}$ \\
\hline Relation du NFP avec les organismes communautaires & $\begin{array}{l}\text { Les ISP sont considérées comme une ressource importante pour connecter leurs clientes avec les } \\
\text { services communautaires. } \\
\text { La participation du NFP permet aux professionnels de la SAE de clore plusieurs dossiers en toute } \\
\text { confiance. } \\
\text { Les ISP jouent un rôle prépondérant dans l'élaboration du plan de sécurité avec les professionnels de } \\
\text { la SAE et la famille. }\end{array}$ \\
\hline
\end{tabular}

Abréviations : ISP, infirmière de la santé publique; NFP, Nurse-Family Partnership.

relation de travail un aperçu des responsabilités du professionnel de la SAE et celles de l'ISP, (2) définir et communiquer clairement les objectifs de chaque professionnel pour le travail auprès de la famille et (3) organiser des rencontres avec la famille pour discuter de la manière d'améliorer le soutien. La collaboration interorganisationnelle figure parmi les stratégies permettant d'éviter le chevauchement des services ${ }^{31}$.

Lors de la phase I, les fournisseurs de soins communautaires avaient également mentionné qu'il était difficile au départ de faire la distinction entre les services de maternité à domicile déjà en place et le NFP. Après avoir travaillé avec les ISP du NFP, ils en sont venus à aimer l'approche du NFP, qui insiste, auprès des jeunes mères défavorisées qui ont un premier enfant, sur les fondements du développement de l'enfant. Lors de la phase II, on a constaté que les membres de l'équipe du NFP avaient fait des efforts considérables pour aider les partenaires communautaires à comprendre les recommandations du programme du NFP, en tenant des réunions avec les décideurs à l'échelon provincial et avec d'autres intervenants communautaires. Le NFP a également fait la promotion de la collaboration avec d'autres organismes en formant des alliances interorganisationnelles, par exemple en coordonnant des conseils consultatifs communautaires où les dirigeants et les fournisseurs de soins directs pouvaient établir des objectifs communs ${ }^{32}$. La mise en place d'un conseil consultatif constitue l'un des 18 éléments du modèle du
NFP que les organismes mettent en œuvre dans le cadre de leur engagement envers le programme. $^{14}$

Nous avons pu constater que le NFP, qui a offert à Hamilton des interventions attendues depuis longtemps et efficaces ciblant les nouvelles mères à risque élevé, a été considéré comme comblant une lacune importante en matière de services. Les professionnels de soins de santé et de services sociaux interrogés dans le cadre de notre étude ont considéré que le succès et la viabilité du NFP au sein de la collectivité reposaient sur le partenariat. Ce résultat va dans le sens des études qui rapportent des résultats plus solides lorsque les programmes de visite à domicile sont jumelés à d'autres services d'intervention précoce et à d'autres programmes communautaires de soutien ${ }^{17}$. Les familles défavorisées bénéfi- ciant de services à la petite enfance ont souvent des besoins complexes qui ne peuvent pas être satisfaits convenablement uniquement par un service de visites à domicile. Les services communautaires doivent donc travailler main dans la main pour cibler ces besoins non satisfaits ${ }^{20}$.

Les professionnels de soins de santé et de services sociaux que nous avons interrogés dans le cadre de cette étude ont également souligné l'importance de continuer à offrir du soutien aux clientes après le NFP afin qu'elles puissent réaliser leur plein potentiel en tant que parents. Cette constatation est corroborée par un bref rapport sur le NFP en Écosse ${ }^{33}$.

\section{Points forts et limites de l'étude}

Notre étude comporte plusieurs points forts. Nous avons eu recours à diverses stratégies

TABLEAU 4

Nouvelles constatations et nouvelles idées relevant de la phase II

\begin{tabular}{|c|c|c|}
\hline Thème & Résumé & $\begin{array}{c}\text { Type } \\
\text { d'information }\end{array}$ \\
\hline $\begin{array}{l}\text { Relation du NFP avec } \\
\text { les organismes } \\
\text { communautaires }\end{array}$ & $\begin{array}{l}\text { L'existence d'une longue liste d'attente pour les mères } \\
\text { admissibles au programme constitue actuellement un défi } \\
\text { important pour leur orientation. }\end{array}$ & $\begin{array}{l}\text { Nouvelle } \\
\text { information }\end{array}$ \\
\hline \multirow[t]{2}{*}{$\begin{array}{l}\text { Le NFP en contexte } \\
\text { communautaire }\end{array}$} & $\begin{array}{l}\text { Les connaissances et les observations de l'ISP sur la famille } \\
\text { sont considérées comme cruciales pour l'obtention pour la } \\
\text { SAE de l'information la plus précise sur l'état de la famille. }\end{array}$ & Approfondissement \\
\hline & $\begin{array}{l}\text { En raison des compressions dans divers services de visite à } \\
\text { domicile de la SAE, la pression de l'afflux de référencements } \\
\text { à la SAE qui en découle peut être réduite car une partie des } \\
\text { cas peut également être confiée au NFP. }\end{array}$ & $\begin{array}{l}\text { Nouvelle } \\
\text { information }\end{array}$ \\
\hline
\end{tabular}

Abréviations : ISP, infirmière de la santé publique; NFP, Nurse-Family Partnership. 
pour en assurer la rigueur méthodologique, en particulier la vérification par les membres de l'équipe, le codage en double et l'utilisation d'une expertise rétrospective. De plus, la taille de l'échantillon de la phase I (24 entrevues) a permis la saturation et l'émergence de thèmes. La mise en œuvre d'un modèle d'étude en deux phases répondait aux défis inhérents aux analyses secondaires de données, à savoir une nouvelle analyse de données susceptible de ne pas refléter adéquatement les nouvelles questions de recherche et une durée et une portée des données de l'étude originale limitées ${ }^{25}$. Les catégories et les thèmes dérivés de la phase I (analyse secondaire des entrevues individuelles) ont servi de guide pour l'élaboration des questions d'entrevue pour la phase II.

Notons également quelques limites importantes, relevant surtout de l'échantillonnage pour la phase II. L'échantillon pour cette phase étant extrêmement réduit (4 entrevues), la saturation avec de nouvelles données pour des thèmes additionnels n'a pas pu être atteinte. Bien qu'il soit impossible d'obtenir des conclusions fermes en raison de la taille très limitée de cet échantillon, nous avons relevé certaines caractéristiques demandant un examen plus approfondi. L'augmentation du nombre de clientes pour la phase II afin d'inclure plus de fournisseurs de soins de santé et de services sociaux liés au NFP aurait amélioré la transférabilité des constatations de cette étude. La triangulation, qui fait appel à l'utilisation de méthodes ou de sources de données différentes pour la collecte et l'analyse de données, par exemple au moyen de groupes de discussion ou de bulletins d'information ou encore de comptes rendus de réunions ${ }^{28}$, aurait également été utile.

\section{Facteurs à prendre en considération pour la recherche future}

Depuis plus d'un siècle, les visites à domicile effectuées par les infirmières constituent l'un des fondements de la santé publique. Malgré l'abondance de connaissances portant sur l'importance de la relation thérapeutique entre l'infirmière, la cliente et sa famille, aucune étude ne s'est encore penchée sur la relation entre l'infirmière effectuant les visites à domicile et les fournisseurs de soins communautaires. Nos résultats suggèrent qu'une exploration plus pointue, dans le contexte des visites à domicile, du rôle des fournisseurs de services sociaux et de soins de santé est importante pour comprendre les approches liées aux prestations et à la mise en œuvre des services.

\section{Répercussions}

Les données recueillies dans le cadre des entrevues individuelles permettent de mieux cerner les éléments clés du succès de l'intégration d'un programme à long terme de visites à domicile au réseau déjà en place de services communautaires. En cela, elles participent à la définition de l'efficacité des interventions, l'ECR en cours en ColombieBritannique constituant l'étape suivante de la mise en œuvre du NFP au Canada. Nous avons identifié quant à nous un besoin de collaboration plus solide avec les divers organismes communautaires et la nécessité de surveiller la manière dont leurs rôles sont susceptibles de se chevaucher.

\section{Conclusion}

Les fournisseurs de soins de santé et de services sociaux ont estimé que le NFP constituait un ajout positif aux services communautaires déjà en place pour les nouvelles mères défavorisées. Les ISP étant intervenues dans le cadre du NFP ont été considérées comme jouant un rôle essentiel, par le fait qu'elles mettent ces mères en contact avec les services communautaires, qu'elles les préparent à la maternité et que l'intervention des services de protection de l'enfance n'est alors parfois pas ou plus nécessaire. Les fournisseurs de soins ont également envisagé une collaboration avec le NFP pour former un réseau intégré de services assurant la transition entre services la plus fluide possible.

Il s'agit de la première étude qualitative visant à explorer la réception d'un programme à long terme de visites à domicile du point de vue des fournisseurs de soins de santé et de services sociaux au sein d'une collectivité. Il serait utile d'examiner les perceptions des fournisseurs de soins de la mise en œuvre continue du NFP et de déterminer jusqu'à quel point il est jugé apte à répondre aux besoins de la collectivité.

\section{Remerciements}

Le financement de la recherche originale a été fourni par le Fonds de recherche sur les soins infirmiers du ministère de la Santé et des Soins de longue durée de l'Ontario et par le Centre d'excellence provincial en santé mentale des enfants et des adolescents au Centre hospitalier pour enfants de l'Est de l'Ontario. Nous désirons témoigner de notre reconnaissance envers les fournisseurs de soins communautaires qui ont participé à l'étude originale (phase I) et ceux qui ont été interrogés pour cette étude (phase II). La $\mathrm{D}^{\text {re }}$ Harriet MacMillan bénéficie du soutien de la Chaire de pédopsychiatrie Chedoke Health. Nous tenons à remercier spécialement la $\mathrm{D}^{\text {re }}$ Masako Tanaka pour son rôle de deuxième codeur, qui a permis d'en venir à un accord entre codeurs.

\section{Références}

1. McCormick MC, Brooks-Gunn J, Shorter T, Holmes JH, Wallace CY, Heagerty MC. Factors associated with smoking in low-income pregnant women in relationship to birthweight, stressful life events, social support, health behaviors and mental distress. J Clin Epidemiol. 1990;43(5):441-448.

2. Sable MR, Wilkinson DS. Impact of perceived stress, major life events and pregnancy attitudes on low birth weight. Fam Plann Perspect. 2000;32:288-294.

3. Issel LM, Forrestal SG, Slaughter J, Wiencrot A, Handler A. A review of prenatal home-visiting effectiveness for improving birth outcomes. J Obstet Gynecol Neonatal Nurs. 2001;40(2):157-165.

4. Gomby DS, Larson CS, Lewit EM, Berman RE. Home visiting: analysis and recommendations. Future Child. 1993;3(3):6-22.

5. Gates R, Nickel D, Muhajarine N, Evaluation Research Team. The effectiveness of home visitation interventions similar to KidsFirst, Saskatchewan: a focused literature review [Internet]. Saskatoon (SK) : Early Learning and Child Care Branch, Saskatchewan Ministry of Education; 2010 [consulté le 23 juin 2014]. PDF téléchargeable à partir du lien : http://kidskan.ca/wp-content/uploads/ 2013/05/3a-Home-Visiting-Lit-Review-KidsFirst-Evaluation-2009.pdf. 
6. Bronfenbrenner U. Ecology of human development: experiments by nature and design. Cambridge (MA) : Harvard University Press; 2005.

7. Bandura A. Self-efficacy: toward a unifying theory of behavioral change. Psychol Rev. 1977;84(2):191-215.

8. Bowlby J. Attachment and loss: Attachment. London (UK) : Hogarth Press and the Institute of Psycho-Analysis; 1969.

9. Olds DL, Eckenrode J, Henderson CR, et al. Long-term effects of home visitation on maternal life course and child abuse and neglect. Fifteen-year follow-up of a randomized trial. JAMA. 1997;278(8) 637-643.

10. Olds D, Robinson J, Pettit L et collab. Effects of home visits by paraprofessionals and by nurses: age 4 follow-up results of a randomized trial. Pediatrics. 2004;114(6): 1560-1568.

11. Olds DL, Kitzman H, Cole RE, Hanks CA, Arcoleo KJ, Anson EA et collab. Enduring effects of prenatal and infancy home visiting by nurses on maternal life course and government spending: follow-up of a randomized trial among children at age 12 years. Arch Pediatr Adolesc Med. 2010;164(5):419-424.

12. Gomby DS. Home visitation in 2005: outcomes for children and parents. Invest in Kids working paper no. 7. Washington (DC): 2005 [consulté le 2 mai 2014]. PDF téléchargeable à partir du lien : wvpartners. org/docs/report_ivk_gomby_2005.pdf

13. Howard KS, Brooks-Gunn J. The role of home-visiting programs in preventing child abuse and neglect. Future Child. 2009;19(2): 119-146.

14. Nurse-Family Partnership. Implementation overview and planning: a guide for prospective Nurse-Family Partnership implementing agencies. Denver (CO) : NurseFamily Partnership; 2010 PDF téléchargeable à partir du lien: http://www.nursefamilypart nership.org/assets/PDF/Policy/HV-Funding-Guidance/NFP_Overview_Planning

15. Nurse-Family Partnership. Pregnancy assistances for first-time moms [Internet]. Denver (CO) : Nurse-Family Partnership; 2011 [consulté le 15 juin 2014]. Consultable à la page : http://www.nursefamilypartnership. org/first-time-moms
16. Hicks D, Larson C, Nelson C, Olds DL, Johnston $\mathrm{E}$. The influence of collaboration on program outcomes: The Colorado Nurse-Family Partnership. Eval Rev. 2008;32(5):453-477.

17. Daro D. Home visitation: assessing progress, managing expectations. Chicago (IL) : Ounce of Prevention Fund; 2006. Joint publication of the Chapin Hall Center for Children. PDF téléchargeable à partir du lien: http://www. chapinhall.org/sites/default/files/old_reports/ 323.pdf

18. Daro D, Cohn-Donnelly A (dir.). Child abuse prevention: accomplishments and challenges. APSAC handbook on child maltreatment ( $2^{\text {nd }}$ ed). Newbury Park (CA) : Sage Publications; 2001.

19. Schumacher R, Hamm K, Goldstein A, Lonbardi J. Starting off right: promoting child development from birth in state early care and education initiatives [Internet]. Washington (DC) : Centre for Law and Social Policy; 2006 [consulté le 2 mai 2014]. Consultable à la page : http://www.clasp. org/publications/startingoffright.html

20. Council on Child and Adolescent Health. The role of home-visitation programs in improving health outcomes for children and families. Pediatrics. 1998;123(2):486-489.

21. Jack SM, Busser D, Sheehan D, Gonzalez A, Zwygers EJ, MacMillan HL. Adaptation and implementation of the Nurse-Family Partnership in Canada. Can J Public Health. 2012;103(7):eS42-eS48.

22. Jack SM, MacMillan HL. Adaptation and evaluation of the Nurse-Family Partnership in Canada [Internet]. The Hague (ND) : Early Childhood Matters; 2014 [consulté le 15 juin 2014]. Consultable à la page : http:// earlychildhoodmagazine.org/adaptation-andevaluation-of-the-nurse-family-partnershipin-canada/

23. Dmytryshyn AL, Jack SM, Ballantyne M, Wahoush O, MacMillan HL. Long-term home visiting with vulnerable young mothers: an interpretive description of the impact on public health nurses. BMC Nursing. 2015; 14:12. doi:10.1186/s12912-015-0061-2.

24. Landy CK, Jack SM, Wahoush O, Sheehan D, MacMillan HL; NFP Hamilton Research Team. Mothers' experiences in the Nurse-Family Partnership program: A qualitative case study. BMC Nursing. 2012;11:15. doi:10.1186/14726955-11-15.
25. Rew L, Koniak-Griffin D, Lewis MA, Miles M, O'Sullivan A. Secondary data analysis: new perspective for adolescent research. Nurs Outlook. 2000;48(5):223-229.

26. NVivo qualitative data analysis software, version 10. Doncaster (VC): QSR International Pty Ltd; 2013.

27. Hsieh HF, Shannon SE. Three approaches to qualitative content analysis. Qual Health Res. 2005;15(9):1277-1288.

28. Lincoln YS, Guba EG. Naturalistic inquiry. Newbury Park (Californie) : Sage Publications.

29. Child and Family Services Act, RSO 1990, c C.11. Consultable à la page. http://www. canlii.org/fr/on/legis/lois/lro-1990-c-c11/ 97652/lro-1990-c-c11.html

30. Landy CK, Jack SM, Wahoush O, Sheehan D, MacMillan HL, NFP Hamilton Research Team. Mothers' experiences in the NurseFamily Partnership program: A qualitative case study. BMC Nurs. 2012;11:15.

31. Peterson N. Interagency collaboration under Part H: the key to comprehensive, multidisciplinary, coordinated infant/toddler intervention. J Early Interv. 2003;15(1):89-105.

32. Foster-Fishman PG, Salem DA, Allen NA, Fahrbach K. Facilitating interorganizational collaboration: the contributions of interorganizational alliances. Am J Community Psychol. 2001;29(6):875-905.

33. Trotter G. Family Nurse Partnership programme in Scotland: an update [Internet]. Community Pract. 2012 [consulté le 15 juin 2014]. Consultable à la page: http://www. readperiodicals.com/201208/2731312821.html 